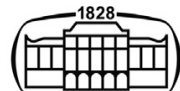

AKADÉMIAI KIADÓ

UNIVERSITY of DEBRECEN

\section{International Review of Applied Sciences and Engineering}

$12(2021)$ 1, 76-85

Dol:

$10.1556 / 1848.2020 .00152$

(c) 2020 The Author(s)

\section{ORIGINAL RESEARCH} PAPER

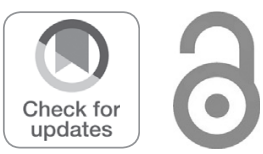

\title{
Development of ECG measurement and processing system in LabVIEW environment
}

\author{
Dávid Dezső ${ }^{1}$, Gyula Győri ${ }^{2}$ and Géza Husi ${ }^{1 *}$ \\ ${ }^{1}$ Mechatronics Department, Faculty of Engineering, University of Debrecen, Debrecen, Hungary \\ ${ }^{2}$ Pharmaflight International Science and Service Center Private Limited Company, Debrecen, Hungary
}

Received: July 07, 2020 • Accepted: November 18, 2020

Published online: December 12, 2020

\begin{abstract}
In this project an ECG measurement and wave identification system was made. The measuring system hardware is based on a Texas Instruments ADS1298ECG amplifier and analog-to-digital converter board. The measurement and processing software was created in LabVIEW environment using the built-in functions of the Biomedical toolkit. Initially, the theory behind heartbeat and its effect on the skin surface potential are presented. Then the measurement techniques of these are described. The research group provide information on the mathematical background of how the ECG curve is processed and the waves are identified. The HRV analysis, the statistical analysis of identified $\mathrm{R}$ waves are described. Subsequently, publications dealing with electrocardiographic examinations in various fields will be presented. Then an overview of the specifications of the amplifiers used in this work and the capabilities of their original software are given. The final device structure is presented. The system validation process and the properties of the reference devices are illustrated.
\end{abstract}

\section{KEYWORDS}

electrocardiography, EKG, ECG, ADC, LabVIEW, HRV, PQRST, ADS1298

\section{INTRODUCTION}

From the outset, the primary goals of technological development have been to extend human life and improve quality. Increasing life expectancy requires not only new drugs and treatments, but also the development of diagnostic systems. With such tools, teams of medical experts measure various vital parameters and physiological values. However, not only doctors but engineers are also needed to build such systems. This requires close cooperation between two very different disciplines, which is a major challenge. For the past year and a half, our research group has been fortunate enough to participate in such a project. Our goal was to integrate the complex study developed by Dr. Máté Petrekanits and his team into a complex measurement system. The purpose of the measurement system is to determine the fatigue, stress levels and overall ability of airplane and helicopter pilots and air traffic controllers to perform their job. This requires simultaneous ECG (electrocardiography), EEG (electroencephalography), skin temperature, muscle oxygenation and vascular stiffness tests at rest. As part of this was given the task of developing the hardware and software for the electrocardiographic part of the examination. The first part of the project is to review the technical details of the ECG amplifier used for testing and initial measurement, and then to present a higher level board embedded in the final system. The research group describes the LabVIEW development environment used to create the software and the SPI-USB board used for communication. This paper is part of larger research project, further results will be published in $[1,2]$.

"Corresponding author.

E-mail: husigeza@eng.unideb.hu

\section{THEORY OF ELECTROCARDIOGRAPHY}

The ECG is the complex uptake of all action potentials generated by myocardial nodes and cells. Each wave or segment of the ECG corresponds to a specific event in the electrical cycle 
of the heart. When the atrium is filled with blood, the SA node sends a signal, the electrical signals spread throughout the atrium and depolarize it. This is represented by the $\mathrm{P}$ wave on the ECG. Atrial contraction, or atrial systole, approx starts $100 \mathrm{~ms}$ after the start of the P wave. The P-Q segment denotes the period during which the signals travel from node SA to node AV. The QRS complex represents the initiation of the AV node signal and represents ventricular depolarization: The $\mathrm{Q}$ wave corresponds to depolarization of the interventional septum. The $\mathrm{R}$ wave is produced by depolarization of the main mass of the chambers. The $S$ wave represents the last phase of ventricular depolarization at the base of the heart. Atrial repolarization also occurs at this time, but the signal is obscured by the large QRS complex. The S-T segment reflects the pause in myocardial action potential. In this case, the chambers contract and pump blood. The $\mathrm{T}$ wave represents ventricular repolarization just before ventricular relaxation or diastole. The cycle is repeated with every heartbeat. During this work other references are processed [4-6] (Fig. 1).

\subsection{Theory of wave identification [7]}

The first step in identifying the $\mathrm{R}$ peak is to preprocess the signal. This requires the identification of the QRS complex, which is carried out using the first and second derivatives. After that, even a signal smoothing is performed with bandpass. Preprocessing is followed by a Hilbert transformation of the signal. The Hilbert transform is one of the most important and common transformations used to detect the QRS complex and the $\mathrm{R}$ wave. The Hilbert transform of the real function $f(t)$ is as follows:

$$
H\{f(t)\}=-\frac{1}{\pi} \int_{-\infty}^{+\infty} f(\tau) \frac{d \tau}{\tau-t} * f(t)
$$

The Hilbert transformation can be defined as the convolution between functions and " $-1 / \pi t$ ". So if we take the definition of the Fourier transformation, we get:

$$
F\{H\{f(t)\}\}=j \operatorname{sgn}(\omega) F(\omega),
$$

where sgn is a signum function. As a result of this transformation, the points intersecting zero become dominant vertices. Adaptive threshold technique ends with peak identification. In this technique, we define a threshold pair of ULT ("Up Limited Threshold") and DLT ("Down Limited Threshold"), where $T H_{m+1}$ and $T H_{f+1}$ are the values of the general threshold, $\mathrm{TH}_{m}$ and $T H_{f}$ the initial values of the threshold. The delta is the difference between the two.

$$
\begin{gathered}
T H_{m+1}=T H_{m}-W_{m} \Delta \\
T H_{f+1}=T H_{f}-W_{f} \Delta \\
\Delta=\left|T H_{m}-T H_{f}\right|
\end{gathered}
$$

The parameters $W_{f}$ and $W_{m}$ are factors of the error mass, which show different values for the number of error detected peaks in each section. Following this step, the output is a waveform consisting of separate dominant vertices which accurately represent the location of the peak of the $\mathrm{R}$ wave.

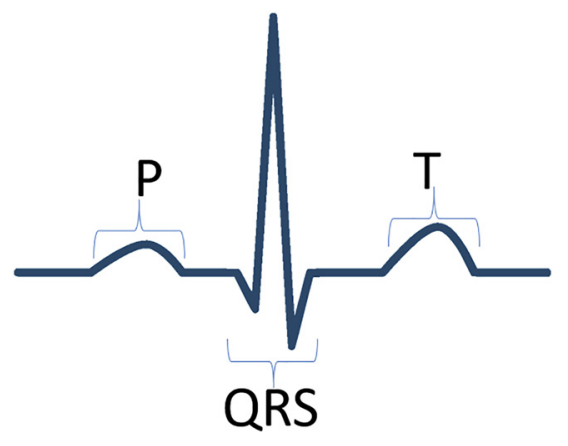

Fig. 1. ECG wave structure [3]

\subsection{ECG leads}

The 12-lead ECG is so called because it provides twelve ECG signals. It uses a reference electrode placed on the right foot (RL) and nine exploratory electrodes: three limb electrodes are placed on the right arm (RA), the left arm (LA) and the left leg (LL), and six electrodes are placed on the trunk near the heart. The position of the electrodes and the signals that can be recorded from the six electrodes above the torso are called precordial leads and are also referred to simply as "thoracic" leads or V1 to V6 wires, while limb signals are called cardinal Einthoven leads, also called LEADs I, II, and III, or simply "limbs". For chest signals, the potential measured at a given point is relative to a central potential value generated inside the amplifier. It is called WCT, or Wilson Central Terminal. More than three augmented signals exist. These could be get from the limb signals by calculation, similarly to the V1-V6 signals these are also unipolar [8].

\section{ADS1298ECG AMPLIFIER [9-11]}

The Texas Instruments ADS1298ECG-FE board is used for measuring. But since the tool we needed to communicate with LabVIEW via SPI was not in our possession yet the data was initially collected by its own software, which we saved in .txt file format and processed it after conversion. The ADS1298ECG-FE is used to evaluate the ADS1298 chip for ECG and EEG applications. The ADS1x98ECG-FE is not a reference model for ECG and EEG applications; rather, it aims to accelerate evaluation and system development. The output of ADS1298 produces a raw, unfiltered ECG signal.

Hardware details:

- Configurable for bipolar or unipolar power supply.

- It can be set for internal and external clock and reference via jumper settings.

- Configurable for AC- or DC-switched inputs.

- Configurable for up to 12 standard ECG leads.

- External Right Leg Drive (RLD) Reference (VCC - VEE)/2.

- External Wilson central voltage.

- Easy connection to popular ECG simulators (Fig. 2). 


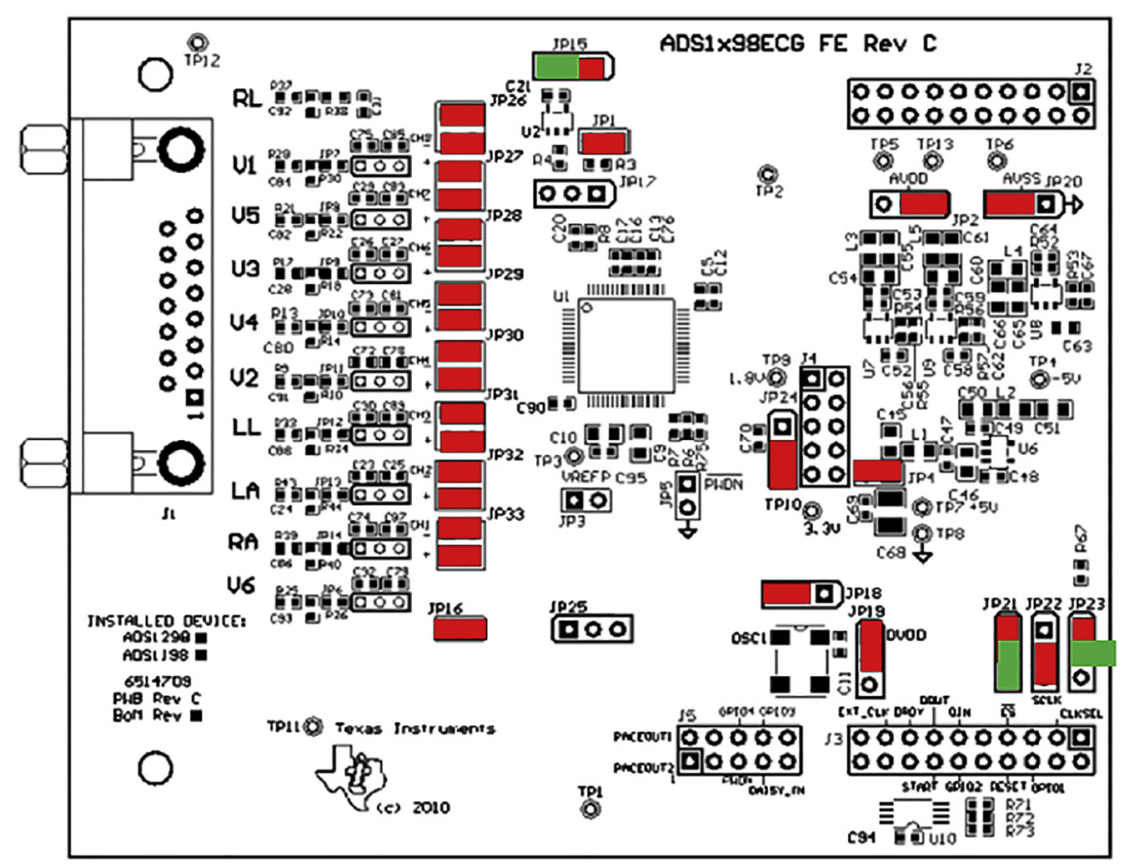

Fig. 2. Original and modified jumper placements (oroginal: red, modified: green)

\section{COMMUNICATION AND DATA COLLECTION}

\subsection{SPI communication}

As mentioned in the previous section, the ADS1298ECG-FE includes a MMB0 communication and acquisition card that allows you to communicate via a USB serial port, but realtime display of all channels was a prerequisite for the project. Based on the tests and the documentation we found, we came to the conclusion that this is not possible with MMB0. So we needed a general-purpose serial-spi converter.

4.1.1. NI USB-8452 [11]. The NI USB-8452 is a high-speed USB device with both I2C (up to $3.3 \mathrm{MHz}$ ) and SPI (up to 50 $\mathrm{MHz}$ ) connections, eight chip selection lines and eight general purpose DIO cables. The NI USB-8452 has a programmable reference voltage that allows communication between I2C, SPI, and DIO at multiple logic levels.

\subsubsection{LabView code}

Measurement initialization. The first step in preparing the measurement is to configure the SPI communication, as the 8,452 can work in several ways. As shown in Fig. 3, as the "Device select" input specifies which port is located on the device, the $3.3 \mathrm{~V}$ signal is given as a constant. This is followed by the clock $(1 \mathrm{kHz})$, polarity (0), and phase (1) settings, and we specify that the amplifier is connected to a zero "chip select" branch. Once you are done with this, direct communication with the amplifier will begin. The basic commands are 16 bit lines of code as shown in Fig. 3.

The following subVI is a two-state electrode register configuration program. As shown in Fig. 4, the "Electrodes" enumeration control allows the operator to select whether the measurement is performed by placing chest plus limb electrodes or by limb only. The figure shows details of how the software sends commands to the amplifier in sequence to modify each channel register. The commands consist of eight bits three times. The difference between the last bit in the last byte means for the amplifier that $\mathrm{CH} 1$ must be turned off and $\mathrm{CH} 2$ must be turned on. With full electrode placement, each channel receives an enabling command. After that, another subVI deals with setting other registers that make resolutions, reference voltages, clock speeds, measurement speeds, and a number of other settings, also in

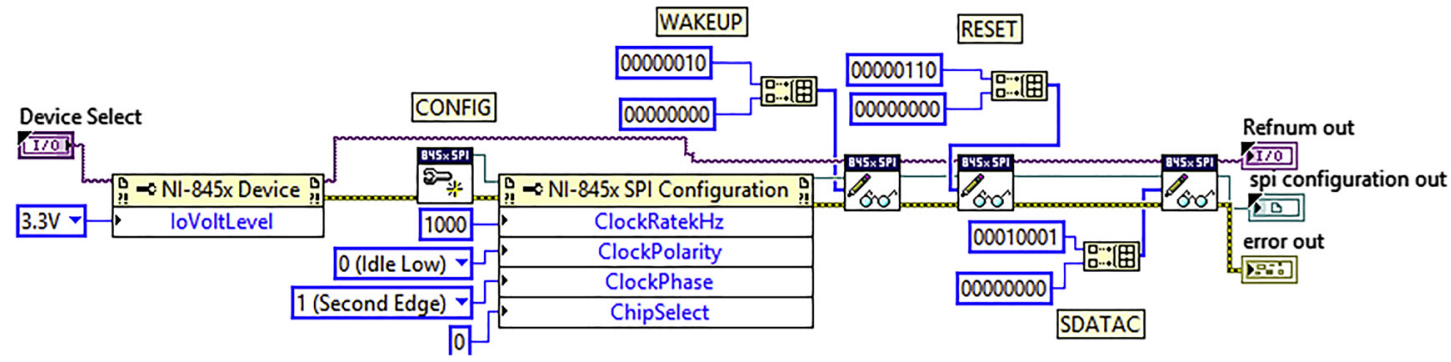

Fig. 3. SPI INIT subVI 


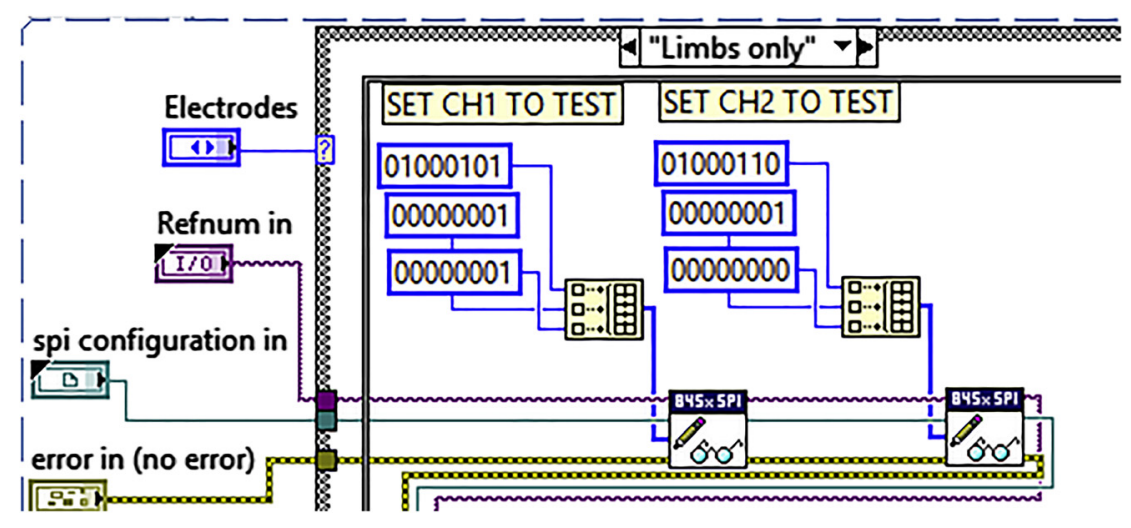

Fig. 4. Electrode register modifications

some cases depending on how many electrodes are used. Finally, all registers are read back by the software for control purposes. This completes the initialization of the measurement.

Data collection and decoding. When the software reaches the "Measurement" state, it starts the "ECG READ" cycle shown in Fig. 5. Here, every $2 \mathrm{~ms}$, the program sends a simple value read command to the amplifier, which responds immediately.

The incoming SPI data is twelve times a 24 bit binary number string to be interpreted.

The bit sequence is encoded with "MSB first" and two's complement. Figure 6 shows the decoding program code. Although the original data is 24 bits, LabVIEW can only store this on 32 bits, so it comes from the scanner, We convert the binary number to a boolean array. We erase the first eight blank values, then separate the sign bit and produce the decoded number depending on its value. Once decomposition and decoding are done, we use the current timestamp to convert the values to "waveform" variables and send them to hidden displays. Each display has a value readout, as shown in Fig. 7, and is positioned in the display cycle. This cycle is designed to allow the operator to choose from any lead that he wants to monitor in real time, without having to load the measuring device with 12 continuous displays. This allows you to choose from a drop-down menu which signal to display on the front panel, on which graph. The drop-down menu values are monitored in a separate cycle with a slower refresh rate to avoid unnecessary workloads on resources.

The remaining measurement time is set in the data reader cycle and the cycle is closed after the time has elapsed.

\subsection{Saving}

After closing the measuring cycle, the enum value of the status selector changes to the save state. The collected data is saved in a ".csv" format with time stamps and columns. The file is named "ECG Channels" and contains the date

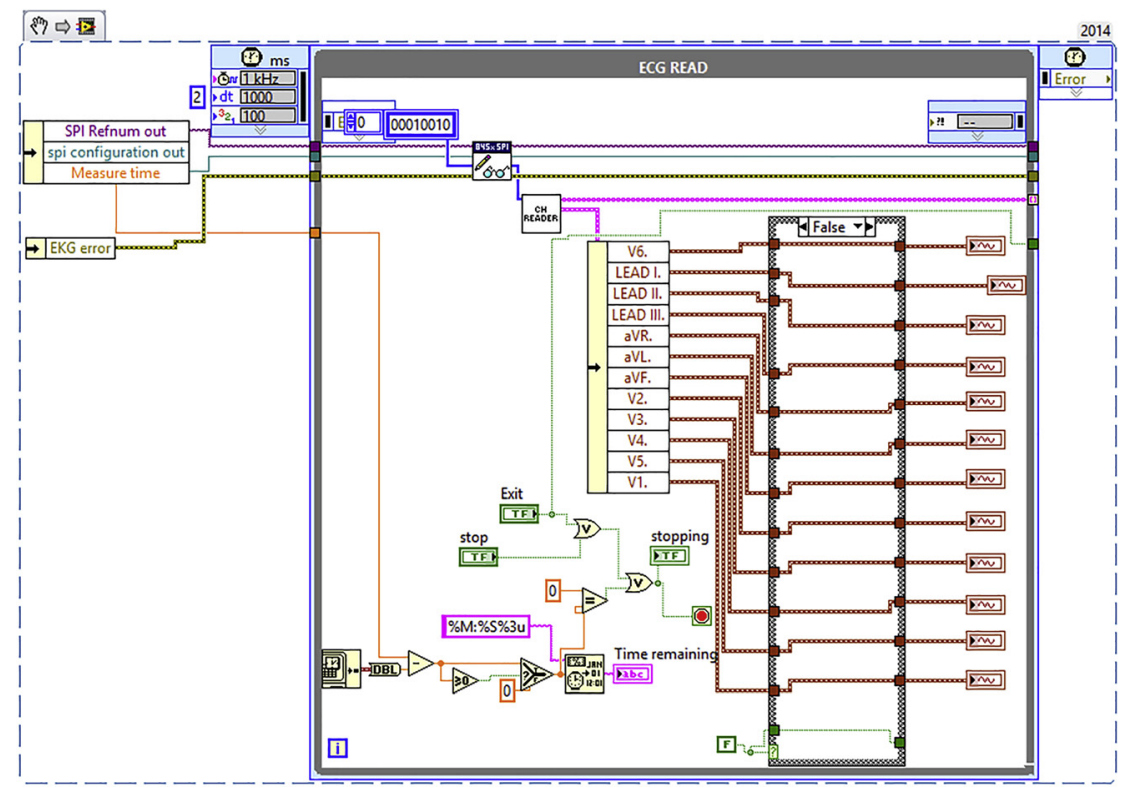

Fig. 5. ECG READ cycle 


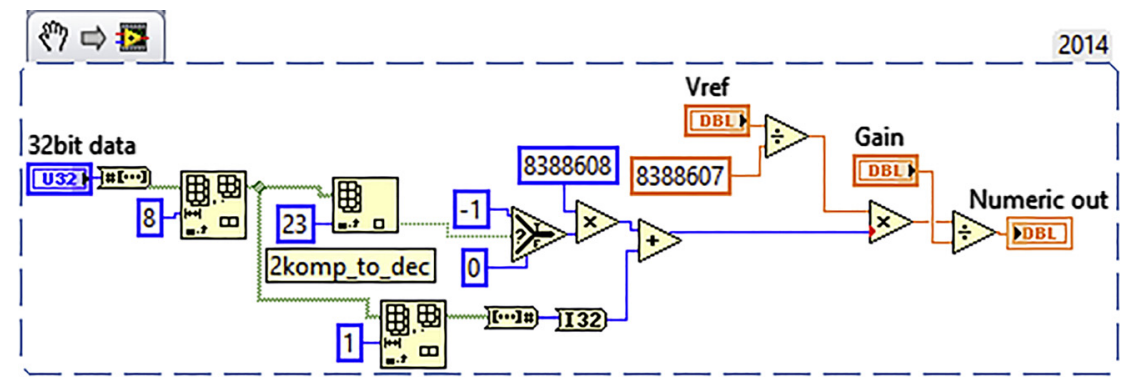

Fig. 6. Decoding program

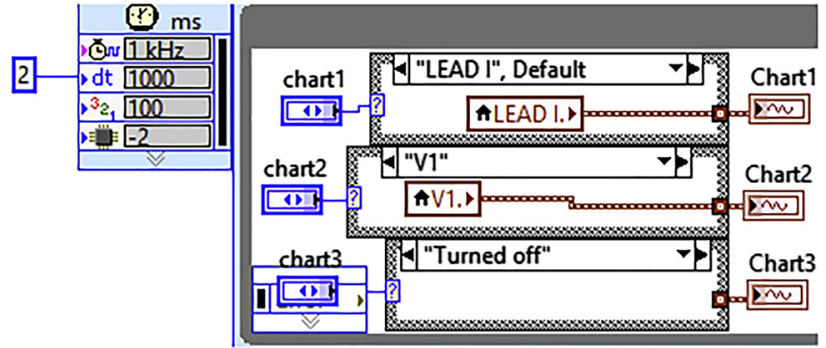

Fig. 7. Display cycle

"ECG_Channels_2019-05-31_02-41-23.csv" with an accuracy of $1 \mathrm{~s}$. After the empty new file is created, we pre-prepare the header so that the complete 13,12 lead plus time, column that comes out of the measuring cycle can be written without modification. At the same time, all the signals from the entire measurement period are displayed on separate graphs on a new tab for the purpose of retrospective examination. After that, the processing section of the software follows.

\section{PROCESSING PROGRAM}

\subsection{Initialization}

The ECG processing phase of the completed software begins with an initialization state. Here, from the main cluster, we extract the string array of 12 channel values collected throughout the measurement, which is converted into a waveform variable and reordered into a new cluster. However, before, it sends them one at a time through a "detrend" filter, this is responsible for removing baseline migration. The data then enters the while loop of this state, where operators have the choice of which derivation they want to process and what parameter settings, and an interval cut option is included. It appears as two graph displays, one showing the shape of one of the leads for the entire 6-minute measurement, and if there were unobtrusive artifacts that you'd rather not process, you can use two cursors to enter a new start and end point, an enlarged view of the cut interval is shown. If the operator clicks the start button, the waves that have already been cut will be saved in a "tdms" file, which will be overwritten during each inter-working backup, which will be needed for wave identification. The interface for these processes is shown in Fig. 8.

\subsection{Wave Identification}

After initialization, the software enters the main ECG processing state. Here, the previously saved .tdms file and the configured parameters are retrieved. On a new display tab, each of the twelve, only 6 at limb measurements, appears on the user interface without baseline wandering. Meanwhile, the biomedical toolkit feature extractor function performs PQRST wave identification on the selected lead. It outputs an array of clusters containing the start and end times of the $\mathrm{P}$, QRS complex, $\mathrm{R}$, and $\mathrm{T}$ waves of each identified heart rate. The processor continues to work with these time indexes. The identification of $\mathrm{P}$ and $\mathrm{T}$ waves is very difficult and it is not possible to determine universal identification parameters, and although the program attempts to process these in all cases, experience shows that a medical review is required to examine these results. In contrast, $\mathrm{R}$-wave identification is a well-defined method, only changing parameters in the case of severe heartbeat abnormalities and disorders, and, of course, examining different leads. As a default setting, physicians have recommended the use of "LEADII" limb lead, with parameters optimized for this purpose as well as test measurements. In order to determine the quality of the wave identification, a coloring view has been added to the processor (Fig. 9).

\subsection{HRV analysis [12]}

HRV analysis is the examination of heart rate variability. HRV analysis is based on the RR interval time chart, the sequence of intervals between successive fiducial ("= reference") points of the QRS complexes in electrocardiography. It should be noted that the R-R interval diagram is actually a sequence of events and is not a continuous signal of equal sampling. This fact is significant, especially when analyzing the frequency range. One of the most important displays with which to start evaluation is the $\mathrm{R}-\mathrm{R}$ diagram on the "spline" tab and the spline function that is fitted to it.

The fitted function is a sixth degree polynomial that approximates the change in mean heart rate over time. This is only possible with sufficient amount of data, so if the tracking function does not fit properly in the chart, it will not be displayed. It is worth starting the evaluation with this 


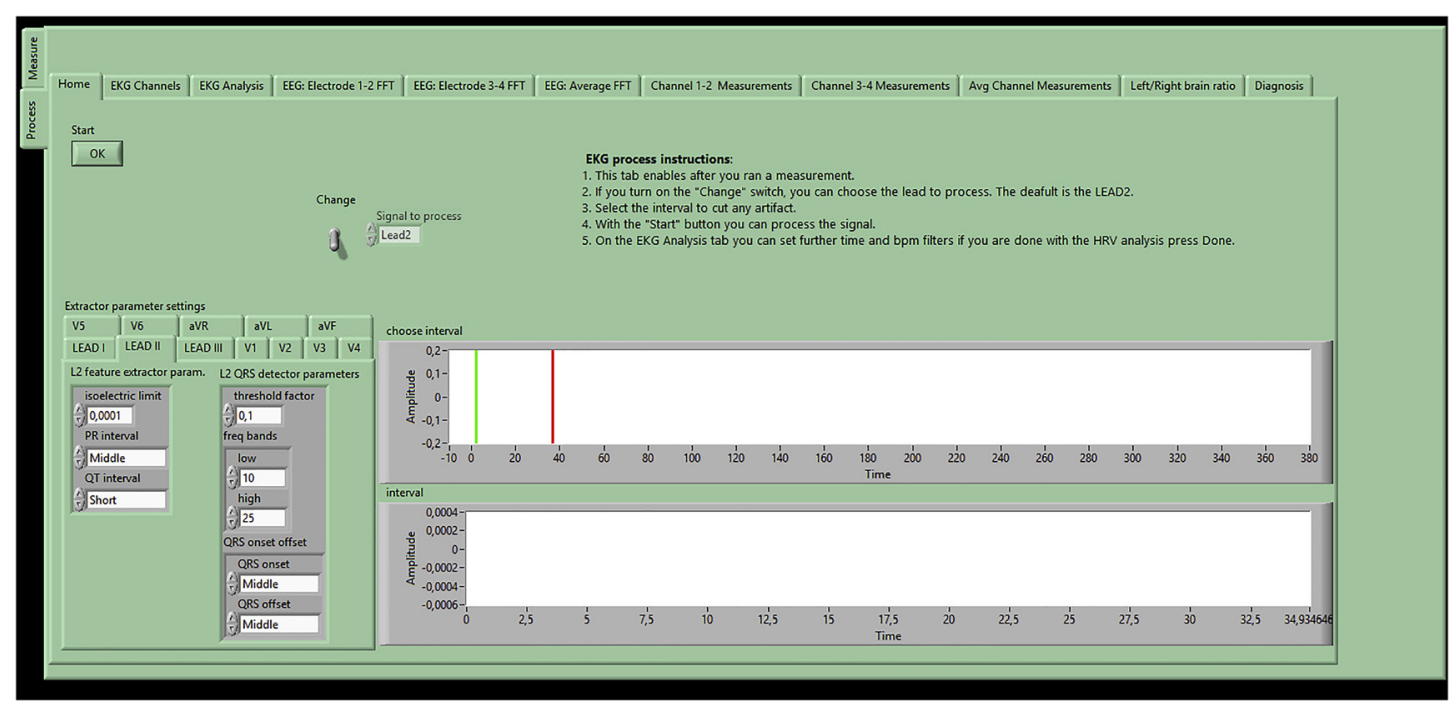

Fig. 8. Init interface

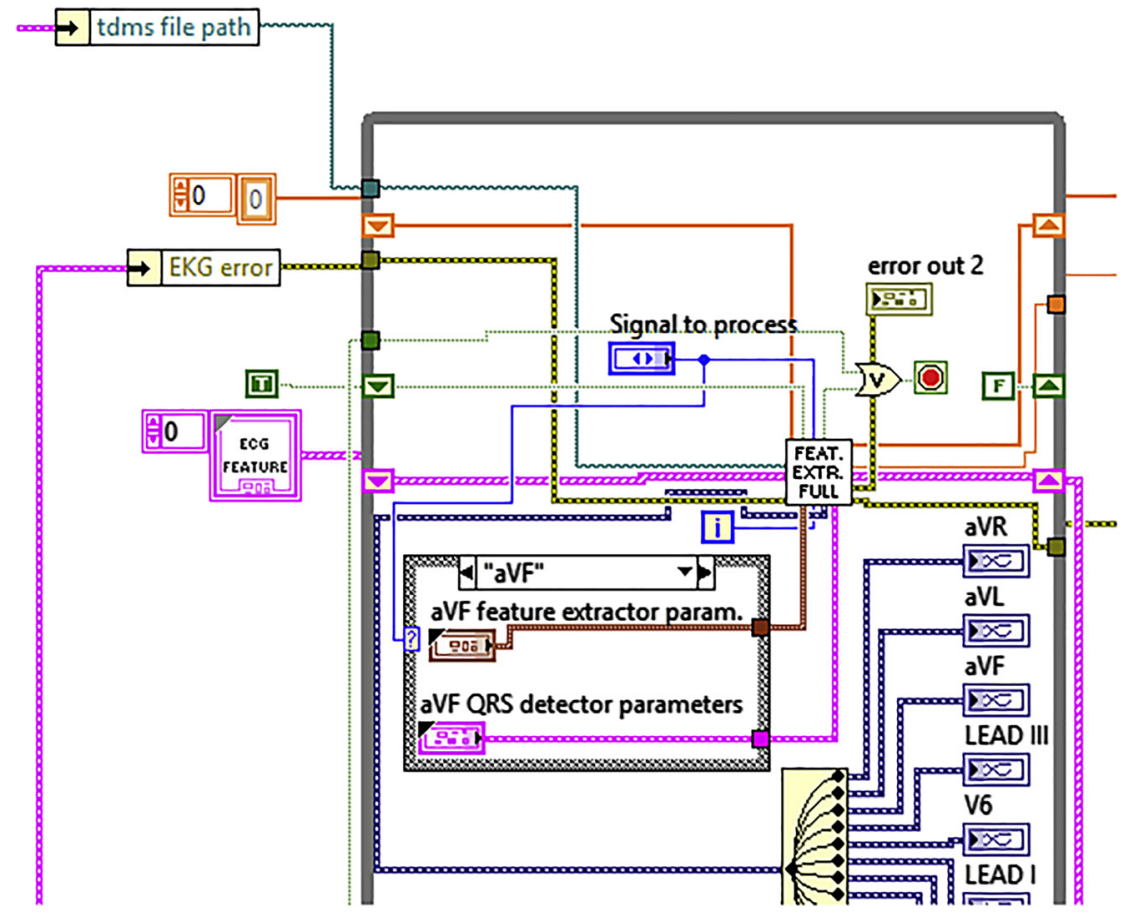

Fig. 9. Wave identification cycle

display because it is clearly displayed here if there was an error in the wave identification. It could be a mistake if an artifact noise was identified as a stroke at this time with extremely high bpm, or if a real stroke could not be identified, then a strikingly low bpm would appear. Since such cases may occur, it is important that you do not have to repeat the entire measurement. For this reason, a secondary and final filter surface is created. At the bottom of Fig. 10 there are two slider interval filters. There is a bpm filter on the left, which is good if you have to cut one or two outliers during the measurement. The right side allows for timely cutting. The switch can be used to decide whether the operator wants to remove an intermediate interval (for example: During measurement, an external effect caused a disturbance in the signal between 120 and $130 \mathrm{~s}$ ) or to process only the appropriate signal between two disturbed signal parts (Fig. 11).

Figure 12 shows the fast Fourier transformation display. As it was read at the beginning of this chapter, this FFT does not need to be interpreted in the same way as the display of a time-constant sampling signal in the frequency domain. This chart translates the rate of change in heart rate into a frequency range. The three colors represent the three specific speeds of this rate of change. The red area shows very small 


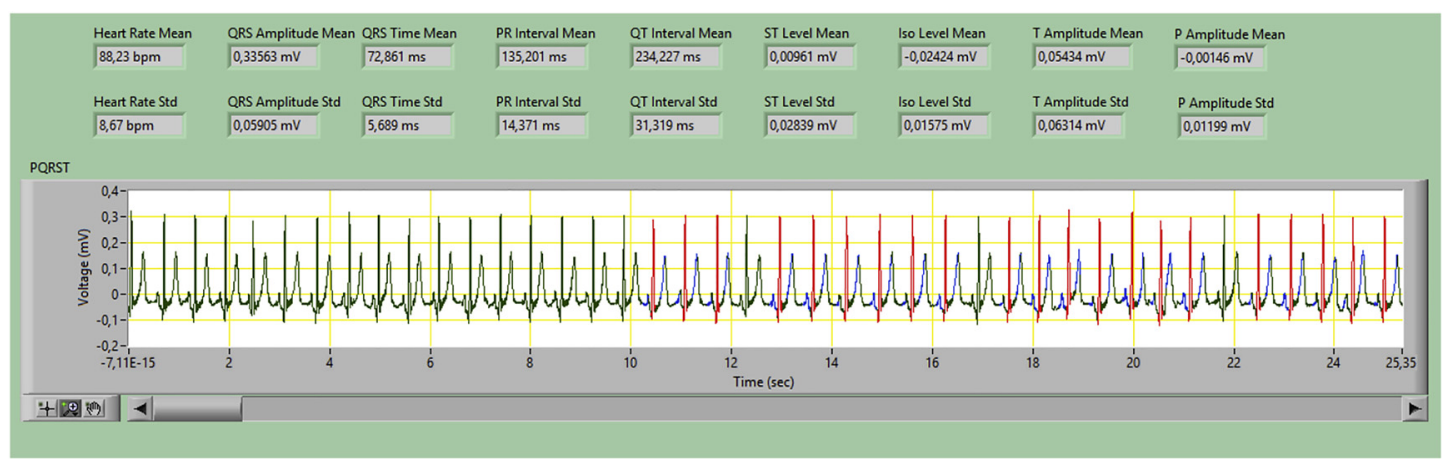

Fig. 10. Colored control display

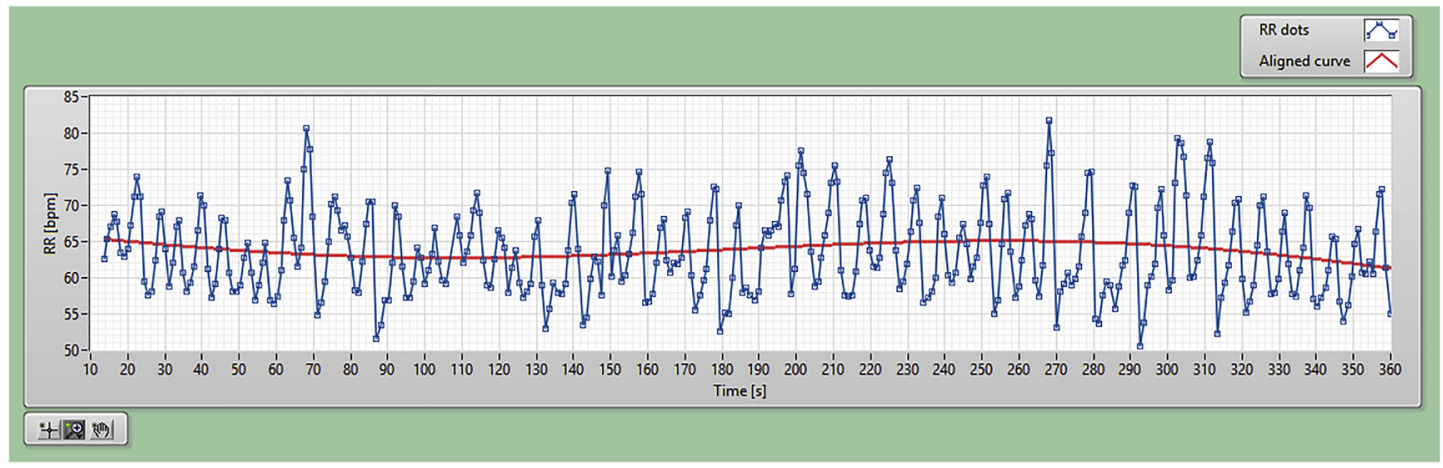

Fig. 11. R-R diagram with spline function

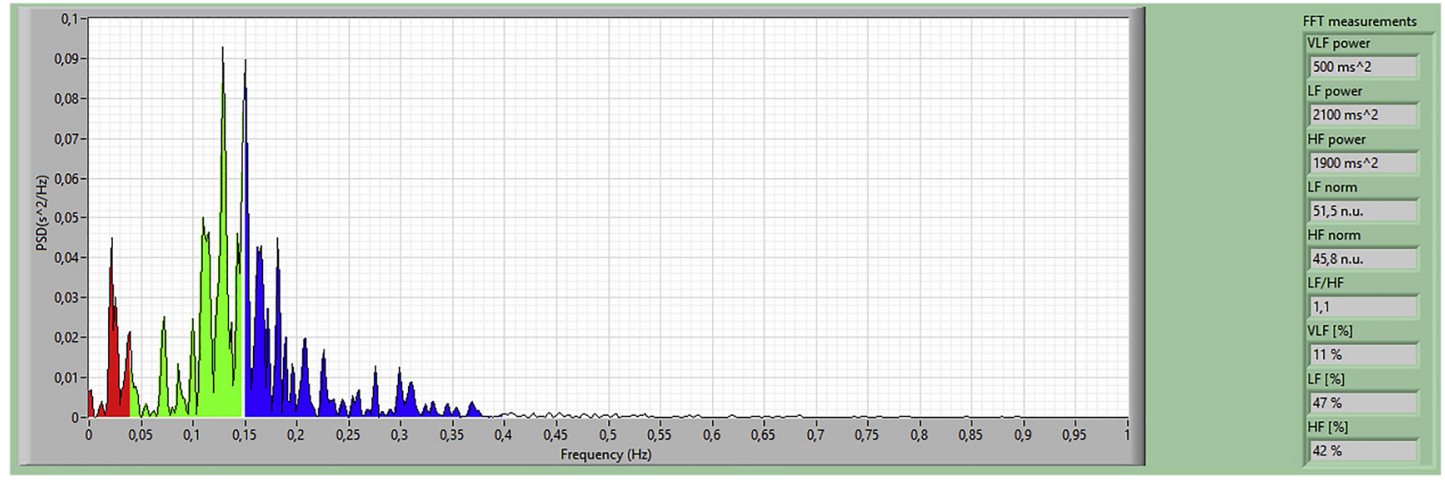

Fig. 12. HRV FFT diagram

changes in VLF (very low frequency). The green area reflects the amount of slow changes in LF ("low frequency"). Finally, blue HF ("high frequency") shows fast pacing changes. For the medical team, the most important metric from this data is the LF/HF dimensionless ratio.

A Poincaré chart for a medically important representation of R-R intervals. As shown in Fig. 12, the intervals, unlike other displays, are not displayed in bpm (beat per minute) but in original seconds. Namely, we represent it as a function of the preceding period as follows: Horizontal axis $\mathrm{RR}_{n}$ (s), vertical axis $\mathrm{RR}_{n+1}(\mathrm{~s})$. This picture gives a good description of the patient's air quality, which the medical team draws from the distribution and symmetry of the set of points (Fig. 13).

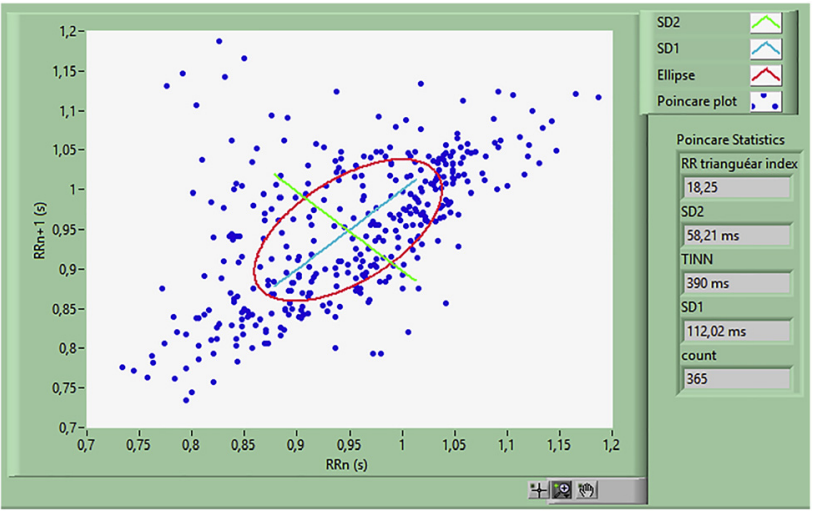

Fig. 13. Poincaré diagram 
The final display of the analysis is a simple histogram. Which indicates the occurrence of a given heart rate by the number of pieces. Figure 14 illustrates the two distinct peaks of the histogram, the reason being that the heart rate is accelerated by inhalation and slows down by exhalation, resulting in an exhalation peak and an inhalation peak. The significant values on this tab are the maximum and minimum heart rate, the difference between them, and the pNN50. PNN50 is a percentage value, counting the number of cases where the absolute difference between consecutive $\mathrm{R}$ intervals was greater than $50 \mathrm{~ms}$, then dividing this number by the total number of $\mathrm{R}$ intervals identified, multiplied by 100 to obtain the percentage value. Once all the necessary filtering has been completed and the analysis found to be satisfactory, clicking on the "Done" button will allow operators to move on to the next part of the software.

\subsection{Diagnosis}

One of the special features of the software is that it not only displays the values used for measured and calculated diagnostics, but also includes an evaluation program section which, based on the given results, draws attention to possible problems if they are outside the optimum section. You do this in the following way. The ECG results are read and then assigned to a group of patients with a diagnosis made by physicians relative to the given thresholds.

\subsection{Riport generation}

Given that this measuring system is intended to be used by a medical team to investigate physically and mentally strenuous people, an important consideration when designing the software was that, once the testing and evaluation had been completed, the test personnel could present some tangible product to the test subject or his/her employer. For this reason, at the end of the software measurement process, we created an automated report generating program. Reporting is located in the state machine just before the "Stop" state, so by the time the operator gets there, he has finalized all the results and processing. We have created a Word template that will be called by the software and filled in using pre-set bookmarks. Which charts and values to include, and the layout and presentation we created with the medical team in series. As the program runs, a new word document pops up and fills in quickly, without the need to open Word in advance. The file is saved by the manager, with the name and location of your choice, and of course you can modify the file.

\section{VALIDATION}

The system was validated by a medical team, in two independent verifications. For the first time, we performed

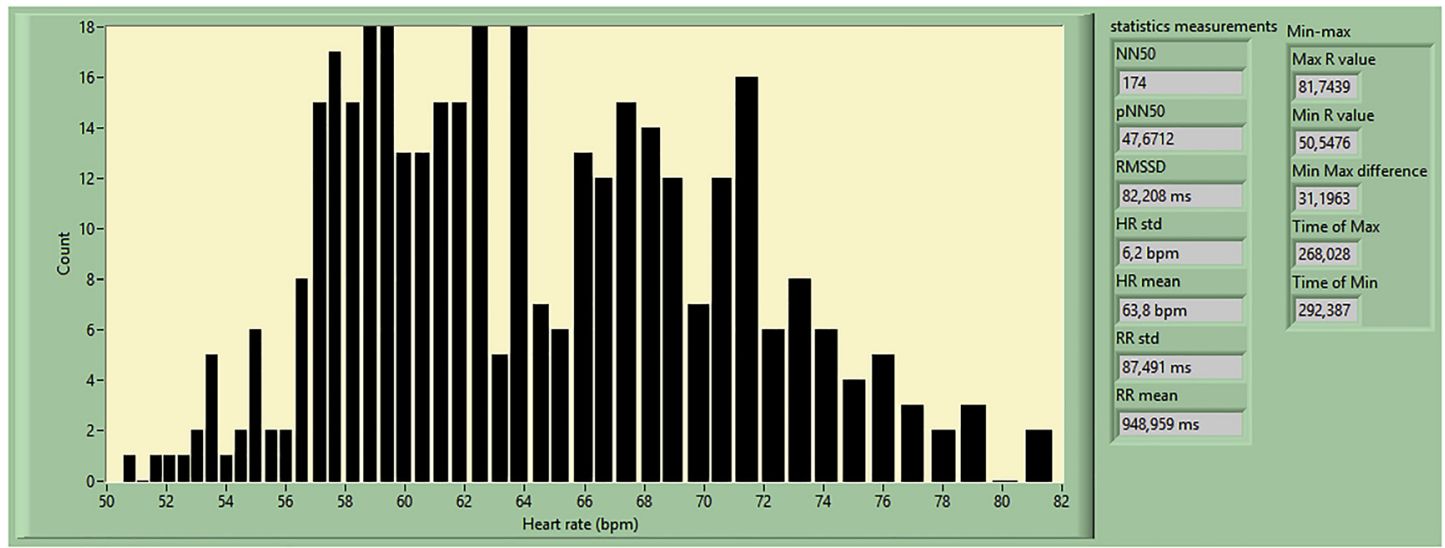

Fig. 14. HRV histogram

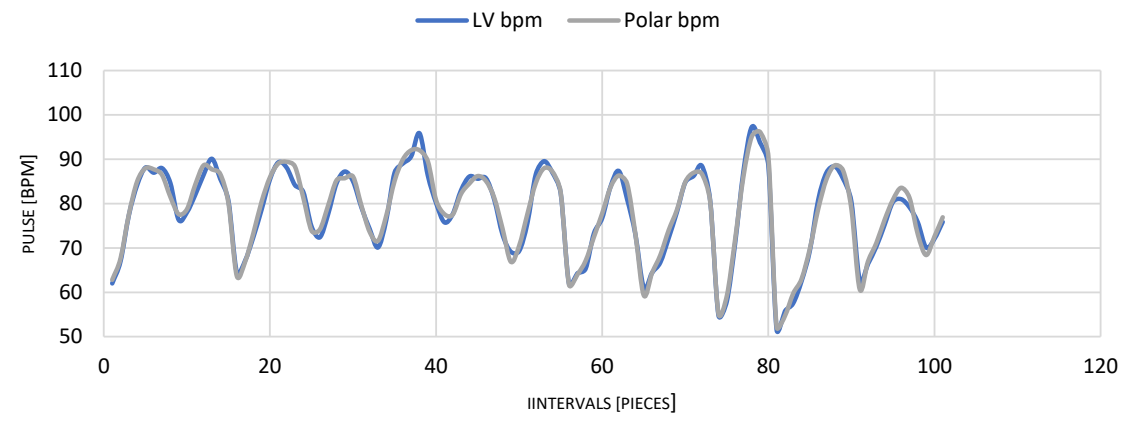

Fig. 15. Result bpm comparison 


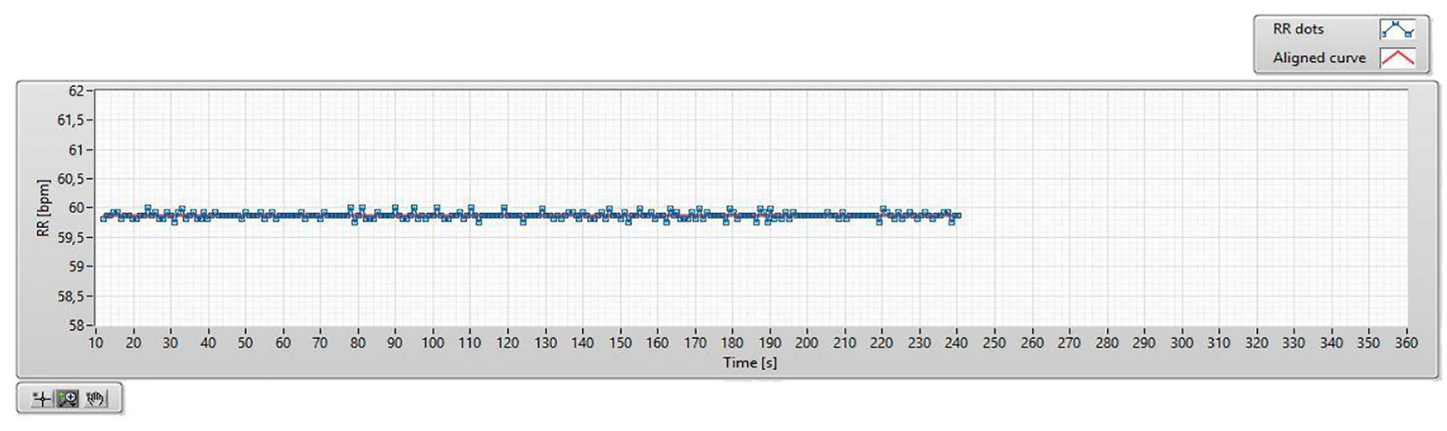

Fig. 16. Constant $60[\mathrm{bpm}]$ result

\begin{tabular}{lcccr}
\hline Value & Unit & $30[\mathrm{bpm}]$ signal & $60[\mathrm{bpm}]$ signal & $90[\mathrm{bpm}]$ signal \\
\hline Min. pulse & $\mathrm{bpm}$ & 29.895366 & 59.760956 & 89.552239 \\
Max. pulse & $\mathrm{bpm}$ & 29.985007 & 60.000000 & 90.225564 \\
Avg. pulse & $\mathrm{bpm}$ & 29.940395 & 59.880814 & 89.908600 \\
Max.-Min. difference & bpm & 0.089641 & 0.239044 & 0.673325 \\
\hline
\end{tabular}

simultaneous measurements on volunteers. For the second time, our software was compared to an ECG generator device. Simultaneous data acquisition was performed with a Polar H2 Activity Chest Sensor [13].

For even greater compliance, we performed measurements with another professional medical device. Dr. Máté Petrekanits provided us with a SIM-02 ECG signal generator [14]. With this tool we have done many complete 12-lead measurements. The peculiarity of such measurements is that it provides a constant heart rate over human subjects, which we can control. This is known as the "input" of the system, that is, what the measurement software should achieve. During the measurements, we set a continuous sinusoidal baseline wander to the signal to test the operation of the baseline wander filtering program. With the device we measured the signals generated at 30,60 and 90 bpm constant values with our own system (Fig. 15).

The meter worked properly. The maximum difference for the ninety heart rate was $0.5 \%$, for the sixty $0.4 \%$ and for the thirty $0.3 \%$, given that the signal generator's technical specification states that it is capable of keeping the heart rate to within $1 \%$ in all cases. Our system measured within error limits (Fig. 16).

\section{SUMMARY}

At the beginning of this article, the reader was introduced to the physics of the heart, and more specifically its properties for use in electrocardiographic diagnostics. Then came the theory of ECG measurements, the positioning of the electrodes and the geometry of the leads. Information is provided on the HAT method used for $\mathrm{R}$ peak identification and on how to analyze the detected pulse in HRV tests. We have selected the necessary hardware for the planned measuring device. Subsequently, a communication interface and data acquisition structure were created for the completed measuring device. We have developed a complex automated ECG signal processing software that can be displayed to the operator to meet any medical requirement. We have created a self-report generating program component that serves as the primary output of the system along with raw voltage data. The finished device was validated against two independent medically recognized devices, an ECG meter and a signal generator.

\section{ACKNOWLEDGMENTS}

The authors would like to thank every medical and technical expert of PHARMAFLIGHT International Science and Service Center PLC for their continuous support during the research activity. The origin of the research topic was initiated by Mr. Gyula Győri.

\section{REFERENCES}

[1] B. G. Barsy, G. Gyori, and P. T. Szemes, "Development of EEG measurement and processing system in labview development environment," Int. Rev. Appl. Sci. Eng., vol. 11, no. 2, pp. 188-96, 2020.

[2] D. Torma, Gy. Győri, and K. Sarvajcz, "Processing and analyzing psychophysiological data using NI DIAdem software," Int. Rev. Appl. Sci. Eng., IRASE, vol. 11, no. 2, pp. 188-96, 2020. https://doi. org/10.1556/1848.2020.20128.

[3] Cardiac Conduction System and Understanding ECG, Animation. [Performance]. Alila Medical Media, 2014.

[4] G. D. Gargiulo, "True unipolar ECG machine for wilson central terminal measurements," BioMed Res. Int. (2015). https://doi.org/ 10.1155/2015/586397.

[5] A. M. Alqudah, A. Albadarneh, I. Abu-Qasmieh, and H. Alquran, "Developing of robust and high accurate ECG beat classification by combining Gaussian mixtures and wavelets features," Australasian Phys. Eng. Sci. Med., vol. 42, no. 1, pp. 149-57, 2019 March 15. 
[6] H. Alquran, A. M. Alqudah, I. Abu-Qasmieh, A. Al-Badarneh, and S. Almashaqbeh, "ECG classification using higher order spectral estimation and deep learning techniques," Neural Network World, vol. 29, no. 4, pp. 207-19, 2019 July 1.

[7] H. Rabbani, M. P. Mahjoob, E. Farahabadi és, and A. Farahabadi, "R peak detection in electrocardiogram signal based on an optimal combination of wavelet transform, hilbert transform, and adaptive thresholding," J. Med. Signals Sensors, \%1. kötet1, \%1. szám2, pp. 91-8, 2011.

[8] www.nottingham.ac.uk, University of Nottingham, [Online]. Available: https://www.nottingham.ac.uk/nursing/practice/ resources/cardiology/function/unipolar_leads. [Hozzáférés dátuma: $01112019]$.
[9] Texas Instruments, ADS1298ECG-FE/ADS1198ECG-FE User's Guide, 2016.

[10] Texas Instruments, ADS129x Low-Power, 8-Channel, 24-Bit Analog Front-End for Biopotential Measurements, 2015.

[11] National Instruments, I2C/SPI NI-845x Hardware and Software Manual, Austin, Texas, 2012.

[12] M. V. Kamath, M. A. Watanabe, A. R. M. Upton, Heart Rate Variability (HRV) Signal Analysis Clinical Applications, CRC Press, 2013.

[13] Polar Electro Oy, Polar H2 Heart Rate Sensor User Manual, Canada, 2014.

[14] L. Farkas és and P. Tóth, SIM-02 Universal Cardiology Simulator, Labtech kft. 